\title{
Contraceptivo oral de emergência: indicações, uso e reações adversas
}

\author{
Emergency contraceptive: indications, use and adverse reactions
}

Contraceptivo oral de emergencia: indicaciones, use y reacciones adversas

Luzilene Gomes de Sousa ${ }^{1}$, Vivian Taís Fernandes Cipriano ${ }^{2 *}$.

\begin{abstract}
RESUMO
Objetivo: Verificar a frequência, faixa etária e outras características populacionais dos usuários do contraceptivo de emergência (CE), avaliar seus efeitos adversos, quais situações motivadoras do uso do medicamento e quais fontes de informação acerca do produto são mais utilizadas. Métodos: Foi realizada pesquisa descritiva durante o período de setembro de 2014 a janeiro de 2018, utilizando as bases de dados SciELO e Pubmed, além de livros relacionados ao tema e os descritores: Anticoncepcional, Contraceptivo de Emergência e Emergency Contraception. Resultados e Conclusão: Apesar das orientações sobre uso correto do CE, os maiores motivos para procura e administração deste medicamento são: insegurança sobre outro método contraceptivo utilizado, não uso do preservativo e/ou rompimento do mesmo e uso incorreto dos anticoncepcionais orais. Adicionalmente, o perfil das usuárias é predominantemente de mulheres com idade entre 16 e 30 anos, sendo 77,8\% com renda inferior a três salários mínimos. Os meios mais utilizados para informações referentes à contracepção emergencial dividem-se em escola/faculdade $65,10 \%$, jornais, revistas e internet $63,60 \%$ e amigos $61,20 \%$. Dentre os efeitos adversos observados relacionados pode-se destacar náuseas, vômitos, sangramento uterino irregular, antecipação ou atraso da menstruação, aumento da sensibilidade mamária, retenção hídrica e cefaleia.
\end{abstract}

Palavras-chave: Contracepção de Emergência, Indicações, Reações Adversas.

\begin{abstract}
Objective: To verify the frequency, age and other population characteristics of emergency contraceptive (EC) users, to evaluate adverse effects of EC use, which reasons for this drug use and which sources of information about the product are most frequently used. Methods: A descriptive study was conducted during the period from September 2014 to January 2018, using the SciELO and Pubmed databases, as well as books related to the subject. As descriptors we had used: Contraceptive, Emergency Contraceptive and Emergency Contraception. Results and Conclusions: Despite the guidelines on correct use of EC, the main reasons for seeking and administering this medicine are: insecurity about another contraceptive method used, non-use of the condom and/ or disruption of the contraceptive method and misuse of oral contraceptives. Additionally, the profile of users is predominantly of women between 16 and 30 years, $77.8 \%$ of whom earn less than three minimum wages. The most used sources for information regarding emergency contraception are divided into school/college $65.10 \%$, newspapers, magazines and internet $63.60 \%$ and friends $61.20 \%$. Among the observed adverse effects it can be observed nausea, vomiting, irregular uterine bleeding and menstruation, increased breast tenderness, water retention and headache.
\end{abstract}

Keywords: Emergency Contraception, Indications, Adverse Reactions.

\section{RESUMEN}

Objetivo: Verificar la frecuencia, edad y otras características poblacionales de los usuarios del anticonceptivo de emergencia ( $\mathrm{AE}$ ), evaluar sus efectos adversos, qué situaciones motivadoras del uso del medicamento y qué fuentes de información sobre el producto se utilizan más. Métodos: Se realizó una investigación

\footnotetext{
${ }^{1}$ Farmacêutica, Faculdade LS Educacional, Brasília - DF.

${ }^{2}$ Farmacêutica, Professora, Doutora e Coordenadora. Curso: Farmácia, faculdade LS Educacional, Brasília DF. *E-mail: vivicipriano@hotmail.com
} 
descriptiva desde septiembre de 2014 hasta de 2018, utilizando las bases de datos SciELO y Pubmed, además de libros relacionados al tema y los descriptores: Anticoncepción, Contraceptivo de Emergencia y Emergency Contraception. Resultados y Conclusión: A pesar de las orientaciones sobre el uso correcto del $\mathrm{AE}$, los mayores motivos para la búsqueda y administración de este medicamento son: inseguridad sobre otro método anticonceptivo utilizado, no uso del preservativo y/o rompimiento del mismo y uso incorrecto de los anticonceptivos orales. Adicionalmente, el perfil de las usuarias es predominantemente de mujeres con edad entre 16 y 30 años, siendo $77,8 \%$ con ingreso inferior a tres salarios mínimos. Los medios más utilizados para informaciones referentes a la contracepción de emergencia se dividen en escuela/universidad 65,10\%, periódicos, revistas e internet $63,60 \%$ y amigos $61,20 \%$. Entre los efectos adversos observados relacionados se pueden destacar náuseas, vómitos, sangrado uterino y menstruación irregular, aumento de la sensibilidad mamaria, retención hídrica y cefalea.

Palabras clave: Contracepción de Emergencia, Indicaciones, Reacciones adversas.

\section{INTRODUÇÃO}

Em 1798, o cientista Thomas Malthus iniciou uma grande discussão ao se opor ao ponto de vista de progresso ilimitado da humanidade. Ele afirmou que se os homens continuassem a "crescer e multiplicar" desordenadamente, não haveriam alimentos e outros compostos necessários à sobrevida humana, em função da sobrecarga do planeta (HENRIQUES A e MALTHUS TR, 2007). Brunton LL et al. (2012) admitiram que Malthus estava certo e, nesse contexto, a paixão entre os sexos persistiu e o poder das populações demonstrou sua grandeza ao ponto dos números populacionais aumentarem e forçarem a capacidade da terra de fornecer alimentos, energia, matérias primas e de absorver os detritos de sua carga humana (BRUNTON LL, et al., 2012).

A ciência médica também tem sua parcela de "culpa" em se tratando de crescimento populacional já que o avanço da saúde pública e terapêutica levaram a um declínio significativo da taxa de mortalidade e aumento na expectativa de vida. Sendo assim, a ciência médica começou também a assumir sua parte na responsabilidade pela superlotação, tornando necessário o desenvolvimento de controle da natalidade humana (ARILHA M e CITELI MT, 2009).

$\mathrm{Na}$ década de 1950 foram realizados estudos clínicos que estabeleceram sucesso com o uso de contraceptivos combinados, formulados a partir de dois fármacos: Noretino e drel-mestranol. Já próximo ao término ano de 1959, o Noretino drel-mestranol tornou-se a primeira pílula de anticoncepcional aprovada pelo FDA (Food and Drug Administration) para ser comercializada nos EUA. Posteriormente, em 1962, ocorreu a aprovação de outra pílula com combinações de Noretidrona com mestranol e em meados 1966 haviam inúmeras formulações utilizando mestranol e estinilestradiol associados. Concomitantemente, foram introduzidas no mercado a minipílula de progestina isolada e as preparações injetáveis de longa duração.

$\mathrm{Na}$ ocasião, muitas mulheres já faziam uso de contraceptivos orais, e como possível consequência desse uso, na década de 1970 ocorreram frequentes relatos de efeitos adversos como: alterações físicas nas mamas, náuseas, cefaleia, retenção de liquido, trombose e diarreia. Tais efeitos adversos por serem comprovadamente dependentes das doses, estimularam modificações nos componentes das fórmulas como também nas suas concentrações (BRUNTON LL, et al., 2012).

No ano de 1980 um anticoncepcional mais confiável, com dosagem hormonal composta por menos da metade das concentrações de estrógeno e progesterona anterior foi alcançado, minimizando as principais queixas relativas aos efeitos colaterais do anticoncepcional oral. Atualmente existe uma variedade de formulações contraceptivas disponíveis incluindo pílulas, injeções, adesivos transdérmicos, implantes subdérmicos, anéis vaginais e DIUs (Dispositivo intrauterino) (BRUNTON LL, et al., 2012).

O Contraceptivo de Emergência (CE) é um medicamento importante para prevenir a gravidez, diferindo dos métodos convencionais como o único utilizado após a relação sexual, tendo sua indicação em situações específicas ou de caráter emergencial como relação sexual sem o uso ou uso inadequado de outro método contraceptivo e violência sexual (BRASIL, 2011; CAVALCANTE MS, 2009; HUTCHISON TA e SHAHAN DR, 2013). Este medicamento hormonal concentrado é composto pela combinação de estrogênio (etinil estradiol) 
e progesterona (levonorgestrel). Descrito em 1972, ficou conhecido como método de Yuspe em homenagem ao médico canadense que o descreveu (SOUZA RA e BRANDÃO ER, 2012).

Desde sua criação, dentre os efeitos adversos observados relacionados ao anticoncepcional de emergência pode-se destacar náuseas, vômitos, sangramento uterino irregular, antecipação ou atraso da menstruação, aumento da sensibilidade mamária, retenção hídrica e cefaleia (FIGUEIREDO R e BASTOS S, 2008). Objetivando minimizar esses efeitos, em 1990 foi desenvolvido então um anticoncepcional de emergência, composto somente por levonorgestrel, o que elevou sua efetividade e reduziu sensivelmente os efeitos adversos (PAIVA SP e BRANDÃO ER, 2012). Desta forma, o contraceptivo oral de emergência é fabricado de duas maneiras: dose dupla, composta por levonorgestrel (progesterona) de $0,75 \mathrm{mg}$ cada e dose unitária composta por levonorgestrel 1,5 mg (BELZER M, 2003).

Conhecida popularmente como "pílula do dia seguinte" por sua utilização pós-relação sexual, ao contrário do que seu nome sugere, não é preciso esperar até o próximo dia para se fazer uso dela, podendo deve ser administrada até 120 horas após a relação sexual desprotegida. (FAÚNDES A et al., 2003). Deve-se também ressaltar que sua eficácia varia em função do tempo entre a exposição sexual e a sua administração, sendo maior eficaz quanto mais próxima a administração da relação sexual (BRASIL, 2011; HUTCHISON TA e SHAHAN DR, 2013).

Por todos os efeitos adversos apresentados e preocupação com a saúde da população, o presente trabalho teve como objetivos verificar a frequência, faixa etária e outras características populacionais dos maiores usuários do $\mathrm{CE}$, avaliar seus efeitos adversos mais presentes, quais motivos que mais motivam o uso do medicamento e quais fontes de informação acerca do produto mais utilizadas.

A presente pesquisa de revisão bibliográfica foi realizada durante o período de 13 de setembro de 2014 a 29 de janeiro de 2018, utilizando como descritores: Anticoncepcional, Contraceptivo de Emergência e Emergency Contraception. As bases de dados acessadas foram SciELO e Pubmed, além de livros relacionados ao tema.

\section{RESULTADOS E DISCUSSÃO}

Segundo o Ministério da Saúde, as indicações para uso da CE são reservadas a situações especiais e excepcionais para prevenção da gravidez inoportuna ou indesejada após relação que, por alguma razão, foi desprotegida. Neste sentido, a administração desse medicamento deve ser reservada à relação sexual sem uso de método anticonceptivo, falha conhecida ou presumida do método em uso de rotina, uso inadequado do contraceptivo ou abuso sexual. Os maiores motivos para o uso da contracepção de emergência pela população são, insegurança no método utilizado, não uso do preservativo e/ou rompimento do mesmo e uso incorreto dos anticoncepcionais orais. Verificou-se também que o uso da CE é aplicado à violência sexual (VELOSO DLC et al., 2014; DREZETT J, 2010; OLIVEIRA WA, 2010; COSTA CA, 2004). Contudo, muitas mulheres fazem o uso da CE como uma contracepção rotineira e não em casos emergenciais como recomendado pelo ministério da saúde, o que é preocupante, pois a CE, além de resultar em diversos efeitos colaterais, não previne doenças sexualmente transmissíveis (DSTs) (ALANO GM, 2012).

Fonteles MMF et al., 2016 afirmam que grande parte das mulheres que fazem uso da contracepção de emergência pertence às classes média e baixa. Sendo assim, $77,8 \%$ tem renda inferior a três salários mínimos, o que pode justificar certa dificuldade para aquisição do medicamento correto, bem como acompanhamento adequado do ginecologista.

Com relação à faixa etária média das usuárias, o perfil é predominantemente de mulheres entre 16 e 30 anos. Esse público utiliza regularmente a $\mathrm{CE}$, geralmente não comprando antecipadamente, mas sim quando acabam de ter tido a relação sexual desprotegida. Normalmente, as mulheres mais jovens tendem à maior utilização do método, pois muitas não querem engravidar por serem jovens e não terem uma vida financeira já estabilizada (ALANO GM, 2012).

Segundo o Ministério da Saúde, as contraindicações para o uso da CE por adolescentes são as mesmas estabelecidas pela Organização Mundial de Saúde (OMS) para o uso por mulheres adultas. É importante que 
se esclareça que não existem contraindicações adicionais ou complementares para o uso da $A E$ nesse grupo etário. Assim, o uso inadequado do CE em adolescentes pode ser tão problemático para esta classe quanto para mulheres mais maduras (ARILHA M e CITELI MT, 2009).

Em pesquisas realizadas com estudantes universitárias, constatou-se que a maioria desse público $(97,1 \%)$ adquire a CE sem nenhuma prescrição médica e que, ao irem às drogarias, nenhuma informação terapêutica ou posológica é passada no momento da compra da medicação. Este fato se torna ainda mais preocupante, pois foi verificado que a maioria das mulheres que fazem uso da CE são as que menos têm informação a respeito do contraceptivo de escolha (ALANO GM, 2012).

Os meios mais utilizados para obtenção dessas informações referentes à contracepção emergencial dividem-se em escola/faculdade $65,10 \%$, jornais, revistas e internet $63,60 \%$ e amigos $61,20 \%$. Esses dados reiteram que a maior parte das informações obtidas é inadequada, o que também contribui para o uso incorreto do CE (ALANO GM, 2012).

Nesse mesmo ínterim, o conhecimento dos estudantes sobre o mecanismo de ação e efeitos colaterais gerados pelo CE é inferior a 50\%, tornando ainda mais visível que muitas mulheres que fazem seu uso não têm informações adequadas (VELOSO DLC et al., 2014; CASTRO JF e RODRIGUES, VMCP, 2009).

Essa informação traz à tona outro dado de extrema importância pública como, a necessidade de melhorar a atuação dos profissionais da área de saúde na orientação adequada de mulheres que procuram o método de $C E$, garantindo assim o seu uso correto, reiterando principalmente sua administração apenas em casos de emergência (ALANO GM, 2012).

A campanha \#vamosdecidirjuntos (2017) pode ser um das poucas respostas para este fato já que a mesma trata-se de uma iniciativa da federação Brasileira de Ginecologia e Obstetrícia (FEBRASGO) que conta com uma pagina virtual (www.vamosdecidirjuntos.com.br) para promover o diálogo entre médicos e pacientes, esclarecer dúvidas sobre vários métodos contraceptivos a partir de vídeos que apresentam orientações de médicos conceituados e assim ampliam discussões sobre a prevenção de gravidez, bem como o método mais indicado para cada perfil (FEBRASGO, 2017).

Preocupado com a questão de natalidade, o Ministério da Saúde, desde 2013, tem facilitado o acesso à pílula do dia seguinte. Os postos de saúde não exigem mais receita médica para distribuir gratuitamente o medicamento desde essa data. Cabe aqui a preocupação com este fato, que ao invés de melhorar a qualidade da informação e/ou medicamento oferecido à paciente, resulta no uso desenfreado e inadequado da medicação por classes desfavorecidas. (VARELLA, M. 2015)

Essa questão pode tornar-se ainda mais preocupante devido à discussão sobre a existência ou não do efeito abortivo pelo uso da CE. Para o Ministério da Saúde e outros pesquisadores, (BATAGLIÃO EML, 2011; CONSTANTINO CF, 2010 e MONTELEONI ML et al., 2006) a pílula não possui esse efeito abortivo e inclusive no ano de 1996 ocorreu a inclusão do medicamento na norma técnica sobre anticoncepção do próprio Ministério, como reflexo do Consórcio Internacional de Anticoncepção de Emergência formado pela OMS juntamente com outras instituições no ano de $1995 \mathrm{com}$ o objetivo de facilitar e consequentemente ampliar o uso e acesso a esse método.

O CE é facilmente adquirido em drogarias e por este motivo é de fundamental importância que o farmacêutico, o qual se insere como linha de frente nesses estabelecimentos, esteja apto a esclarecer eventuais dúvidas. O farmacêutico com todo o seu conhecimento a respeito de mecanismo de ação, farmacodinâmica e farmacocinética exerce papel fundamental para promoção do uso racional de medicamentos, evitando assim a automedicação e possíveis efeitos adversos (ALANO GM, 2012) mais frequentes como: náuseas, em 40 a $50 \%$ dos casos, vômito, em 15 a $20 \%$ além de alterações do ciclo menstrual (FONTELES MMF et al., 2016; ALANO GM, 2012).

Estimativas indicam que, no Brasil, $22,1 \%$ das mulheres adotam a pílula anticoncepcional como método para prevenir a gravidez, seguindo-se do preservativo masculino (12,9\%), injeção contraceptiva (3,5\%) e dispositivo intrauterino - DIU (1,5\%). Os métodos tradicionais (comportamentais) tabelinha e o coito 
interrompido somam 2,4\% (SOUSA 2017; CAVALCANTE MS, 2009). A eficácia do CE pode variar de forma importante em função do tempo entre a relação sexual e sua administração. Segundo a OMS, suas taxas de falhas são de $2 \%$ até 24 horas após relação sexual, 4,1\% entre 25 e 48 horas após relação sexual e 4,7\% entre 49 e 72 horas (VON HERTZEN H, et al., 2002).

\section{CONSIDERAÇÕES FINAIS}

Foi observado que os maiores motivos para procura e administração do CE são: insegurança sobre outro método contraceptivo utilizado, não uso do preservativo e/ou rompimento do mesmo e uso incorreto dos anticoncepcionais orais. O perfil das usuárias é predominantemente de mulheres com idade entre 16 e 30 anos. Dentre os meios de informação farmacoterapêutica apresentados pelos usuários destacam-se escola/faculdade, jornais, revistas, internet e amigos. Adicionalmente, os efeitos adversos mais observados foram náuseas, vômitos, sangramento uterino irregular, antecipação ou atraso da menstruação, aumento da sensibilidade mamária, retenção hídrica e cefaleia.

\section{REFERÊNCIAS}

1. ALANO GM. Conhecimento, consumo e acesso à contracepção de emergência entre mulheres universitárias no sul do Estado de Santa Catarina. Ciência e saúde coletiva, 2012; V.17 n 9 p. 2397-2404.

2. ARILHA M, CITELI MT. Intervenções restritivas ao acesso da Contracepção de Emergência no Brasil (1999-2009): um mapa geopolítico das barreiras aos direitos reprodutivos no país. In: ARILHA, M.; LAPA, T. S.; PISANESCHI, T. C. Contracepção de emergência no Brasil e América Latina: dinâmicas políticas e direitos sexuais e reprodutivos. São Paulo, 2009; p.135-162.

3. BATAGLIÃO EML. Contracepção de Emergência e acadêmicos de Enfermagem. Esc. Anna Nery, Ribeirão Preto, 2011; v.15 n. 2, p284-290.

4. BELZER M. Advanced supply of emergency contraception for adolescent mothers increased utilization without reducing condom or primary contraception use. Journal of Pediatric and Adolescent, Mount Royal, 2003; v.32, n.2, p.122-3.

5. BRUNTON LL, et al. As Bases Farmacológicas da Terapêutica de Goodman e Gilmar. Editora AMGH Ltda. Porto Alegre, 2012. 385p.

6. CASTRO JF, RODRIGUES VMCP. Conhecimentos e atitudes dos jovens face à contracepção de emergência. Revista da Escola de Enfermagem da USP, São Paulo, 2009; v.43, n.4, p.889-94.

7. CAVALCANTE MS. Perfil de utilização de contraceptivo de emergência a partir de um serviço de atendimento farmacêutico de uma rede de farmácias comunitárias, (Mestrado), Universidade Federal do Ceará, Fortaleza, $2009 ; 90$ f.

8. CONSTANTINO CF. Contracepção de emergência e adolescência: Responsabilidade e Ética. Revista Bioética, 2010; v. 18, n. 2, p. 347-61.

9. COSTA CA. Anticoncepção de emergência: a pílula do dia seguinte. Revista a Saúde da Mulher. Rio de Janeiro, 2004.

10. DREZETT J. Contracepção de emergência: normativas, usos, mitos e estigmas. In: ARILHA, M.; LAPAK, T. S.; PISANESCHI, T.C. (Org). Contracepção de emergência no Brasil e América Latina: dinâmicas políticas e direitos sexuais e reprodutivos. São Paulo: Oficina Editorial, 2010; p.63-89.

11. FAÚNDES A, et al. Emergency contraception under attack in Latin America: response of the medical establishment and civil society. Reproductive Health Matters, London, 2007; v.15, n.29, p.130-138.

12. FEBRASGO. 2017. In: FEBRASGO. Disponível em: htpp://www.vamosdecidirjuntos.com.br. Acesso em: 20 de fevereiro de 2018.

13. FIGUEIREDO R, BASTOS S. Contracepção de emergência: atualização, abordagem, adoção e impactos em estratégias de DST/AIDS. São Paulo: Instituto de Saúde, 2008; p. 52.

14. FONTELES MMF, et al. Perfil de utilização de anticoncepcional de emergência em serviços de atendimento farmacêutico de uma rede de farmácias comunitárias. Eletronic journal of pharmacy, 2016; vol. XIII, n. 3, p.131139.

15. HENRIQUES A, Thomas RM. A Teoria MAthusiana. Instituto Superior de Engenharia de Coimbra, junho de 2007.

16. HUTCHISON TA, SHAHAN DR. (EDS): DRUGDEX® System. MICROMEDEX, Inc., Greenwood Village; 2011.

17. MONTELEONI, ML, et al. Anticoncepção de Emergência e a Adolescente. In: CONGRESSO LATINOAMERICANO DE GINECOOBSTETRÍcIA, Rio de Janeiro, 2006; Resumos.

18. OLIVEIRA WA. Fatores associados à informação e à prescrição da pí- lula anticoncepcional de emergência (PAE) por ginecologistas e obstetras brasileiros. Dissertação (Mestrado) - Faculdade de Ciências Médicas. Universidade Estadual de Campinas. Campinas, 2010; $128 \mathrm{p}$.

19. PAIVA SP, BRANDÃO E.R. Contracepção de emergência no contexto das farmácias: revisão crítica de literatura. Physis Revista de Saúde Coletiva, Rio de Janeiro, 2012; v. 22, n. 1, p. 17-34.

20. SOUZA RA, BRANDÃO ER. À sombra do aborto: o debate social sobre a anticoncepção de emergência na mídia impressa brasileira (2005-2009). Interface - Saúde, Educação, Comunicação, Botucatu, 2012; v.16, n.40, p.161-175.

21. VARELLA M, 2015. In: A falta de informação sobre a pílula do dia seguinte. Disponível em: http//:www.drauziovaerlla.uol.com.br. Acesso em: 05 de maior de 2018.

22. VELOSO DLC, et al. Anticoncepção de emergência: conhecimento e atitude de acadêmicos de enfermagem. Revista Gaúcha de Enfermagem, Porto Alegre, 2014; v.35, n.2, p.33-9.

23. VON HERTZEN H, et al. Low dose mifeprostone and two regimes of levonorgestrel for emergency contraception: a WHO multicentre randomized trial. Lancet., [s.I.], 2002; v. 360, p. 1803-1810. 